UCSD-PTH-00-34

IASSNS-HEP-00/88

MIT-CTP-3068

\title{
Radion Stabilization by Brane Matter
}

\author{
Benjamín Grinstein*, Detlef R. Nolte ${ }^{\dagger}$, and Witold Skiba ${ }^{\ddagger}$ \\ * Department of Physics, University of California at San Diego, La Jolla, CA 92093 \\ ${ }^{\dagger}$ Institute for Advanced Study, Princeton, NJ 08540 \\ ${ }^{\ddagger}$ Center for Theoretical Physics, Massachusetts Institute of Technology, Cambridge, MA 02139
}

(December 2000)

\begin{abstract}
We find a static solution to Einstein's field equations on a five-dimensional orbifold with a compact $S_{1} / Z_{2}$ fifth direction and Poincare invariant $3+1$ sections. The solution describes a theory with bulk cosmological constant and 3-branes at the orbifold fixed points which carry matter density and pressure in addition to tension. The radius of the fifth dimension is determined by the matter content of the branes. The ratio of the space and time components of the metric depends on the fifth coordinate. Thus, the speed of propagation of massless fields is path dependent. For example, bulk and brane fields propagate with different speeds.
\end{abstract}

\section{INTRODUCTION}

A solution to the hierarchy problem has been proposed in which the observable universe is a 3-brane at an orbifold fixed point of the non-factorizable geometry

$$
d s^{2}=e^{-2 k|y|} \eta_{\mu \nu} d x^{\mu} d x^{\nu}-d y^{2} .
$$

The orbifold has fixed points at $y=0$ and $y=y_{c}$ where there are branes with tension $V_{0}=12 k$ and $V_{c}=-12 k$, respectively. We will refer to this as the RS model [1]. However, the dynamics does not determine the value of $y_{c}$, leaving it a free parameter. A solution to this so called "radion stabilization problem" has been found by adding a bulk scalar field, that is, one that has five-dimensional dynamics, to the model [2]. Alternative stabilization mechanisms have been proposed in [3].

In this paper we present a static solution of the field equations when the branes carry matter density and pressure in addition to tension. The solution fixes the radius of the

\footnotetext{
*e-mail addresses: bgrinstein@ucsd.edu, nolte@ias.edu, skiba@mit.edu
} 
fifth dimension. To see how this works let's first recall why there is a radion stabilization problem in the RS model. The warp factor in Eq. (1.1), $A=-k|y|$, has a constant slope and, therefore, the jump of the slope across the fixed point is $-2 k$ regardless of where the jump occurs. Now if the warp factor had a non-constant slope $A^{\prime}(y)$, then the jump $-2 A^{\prime}\left(y_{c}\right)$ does depend on $y_{c}$. Therefore the radius of the fifth dimension has to be chosen to accommodate the jump in energy density on the brane.

Adding matter to the basic setup of RS is necessary for a description of cosmology of the model. The cosmology of brane models has been investigated in a number of papers. A general formulation was given in Ref. [4]. The work in Refs. [5 7] is concerned with the cosmology of brane models of the Randall-Sundrum type [四]. These papers recognize that the jump in the warp factors across the branes as implied by Einstein's equations is enough to give the evolution of the scale factor in a FRW description of the cosmology on one brane. Therefore there has been little interest in the behavior of the metric in the bulk, see however Ref. [7]. It turns out that investigating bulk solutions can yield interesting results. The solutions we find exist only for a particular value of brane separation, which is determined by the matter content of the branes. Depending on the kind of matter such solutions can be either stable or unstable under small perturbations. The salient feature of our bulk solutions induced by brane matter, similar to the solutions in Ref. [8], is that the space and time components of the metric are not identical as they are in the RS model without matter. This means that the speed of propagation of massless fields depends on their trajectory. For example, bulk gravitons can propagate at a speed different than bulk photons. Finding out the strength of gravity and masses of bulk and brane fields in such complicated backgrounds is straightforward using the results of Ref. [9].

In Refs. [4] [6] it is found that the scale factor $a$ satisfies

$$
\left(\frac{\dot{a}}{a}\right)^{2}+\left(\frac{\ddot{a}}{a}\right)=\frac{1}{72} V(\rho-3 p)-\frac{1}{36} \rho(\rho+3 p),
$$

where $V, \rho$ and $p$ stand for the tension, matter density and pressure on the brane respectively. We have set the five-dimensional gravitational constant to unity. Our static solution is not in contradiction with this brane equation because the solution demands that the three parameters $V, \rho$ and $p$ are such that the right hand side vanishes.

We present our solution in section $\mathbb{1 1}$. We study non-static solutions as small perturbations to our solution in Sec. III and present our conclusions in section IV.

\section{A STATIC SOLUTION WITH MATTER}

We denote the coordinates of spacetime by $x^{A}, A=0, \ldots, 4$, and often use $t=x^{0}$ and $y=x^{4}$. The fixed points are at $y=0$ and $y=y_{c}$. The class of spherically symmetric metrics we study is parameterized by three functions of $t$ and $y$ only [4]

$$
d s^{2}=G_{A B} d x^{A} d x^{B}=n^{2}(t, y) d t^{2}-a^{2}(t, y) d \vec{x}^{2}-b^{2}(t, y) d y^{2} .
$$

Fixing $y=0\left(y=y_{c}\right)$ we see that the metric gives a flat FRW cosmology on the brane with scale factor $R_{0}\left(t^{\prime}\right)=a\left(t\left(t^{\prime}\right), 0\right)\left(R_{c}\left(t^{\prime}\right)=a\left(t\left(t^{\prime}\right), y_{c}\right)\right)$ where $d t^{\prime}=n(t, 0) d t\left(d t^{\prime}=n\left(t, y_{c}\right) d t\right)$. We will denote by $g_{\mu \nu}$, with $\mu, \nu=0, \ldots, 3$, the induced metric on the brane. 
The action is

$$
S=\int d^{5} x \sqrt{G}[-R-\Lambda]+\int d^{4} x \sqrt{-g}\left[-V_{0}\right]_{y=0}+\int d^{4} x \sqrt{-g}\left[-V_{c}\right]_{y=y_{c}} .
$$

The constants $\Lambda, V_{0}$ and $V_{c}$ represent the cosmological constant in the bulk (5-dimensional space) and on the branes at $y=0$ and $y=y_{c}$, respectively. In addition there is matter density and pressure on the branes, introduced into the field equations directly by their contribution to the energy momentum tensor:

$$
T^{A B}=\tilde{T}^{A B}+\frac{S_{0}^{A B}}{b} \delta(y)+\frac{S_{c}^{A B}}{b} \delta\left(y-y_{c}\right)
$$

where $\tilde{T}^{A B}$ is derived as usual by varying the action with respect to the metric, and $S^{A B}$ are contributions from perfect fluids of density $\rho_{0}$ and $\rho_{c}$ and pressures $p_{0}$ and $p_{c}$ on the branes,

$$
S_{B}^{A}=\operatorname{diag}(\rho,-p,-p,-p, 0) .
$$

Einstein's equations are

$$
R^{A B}-\frac{1}{2} G^{A B} R=\kappa T^{A B}
$$

Here $R^{A B}$ and $R$ are the Ricci tensor and scalar. The gravitational constant is $\kappa$ and we work in units of $\kappa=1$.

For the particular metric (2.1) Einstein's equations are

$3\left[\left(\left(\frac{\dot{a}}{a}\right)^{2}+\frac{\dot{a} \dot{b}}{a b}\right)+\frac{n^{2}}{b^{2}}\left(-\frac{a^{\prime \prime}}{a}-\left(\frac{a^{\prime}}{a}\right)^{2}+\frac{a^{\prime} b^{\prime}}{a b}\right)\right]=\frac{1}{2} n^{2} \Lambda+\delta(y) \frac{n^{2}}{b}\left(\frac{1}{2} V_{0}+\rho_{0}\right)+$

$$
\delta\left(y-y_{c}\right) \frac{n^{2}}{b}\left(\frac{1}{2} V_{c}+\rho_{c}\right)
$$

$$
3\left(\frac{\dot{a} n^{\prime}}{a n}+\frac{a^{\prime} \dot{b}}{a b}-\frac{\dot{a}^{\prime}}{a}\right)=0,
$$

$\frac{a^{2}}{n^{2}}\left(-\frac{\dot{a}^{2}}{a^{2}}-2 \frac{\ddot{a}}{a}+2 \frac{\dot{a} \dot{n}}{a n}-2 \frac{\dot{a} \dot{b}}{a b}-\frac{\ddot{b}}{b}+\frac{\dot{n} \dot{b}}{n b}\right)+\frac{a^{2}}{b^{2}}\left(\frac{a^{\prime 2}}{a^{2}}+2 \frac{a^{\prime \prime}}{a}+2 \frac{a^{\prime} n^{\prime}}{a n}-2 \frac{a^{\prime} b^{\prime}}{a b}+\frac{n^{\prime \prime}}{n}-\frac{n^{\prime} b^{\prime}}{n b}\right)$

$$
=-\frac{a^{2}}{2} \Lambda-\delta(y) \frac{a^{2}}{b}\left(\frac{1}{2} V_{0}-p_{0}\right)-\delta\left(y-y_{c}\right) \frac{a^{2}}{b}\left(\frac{1}{2} V_{c}-p_{c}\right),
$$

$3\left[\frac{b^{2}}{n^{2}}\left(-\frac{\dot{a}^{2}}{a^{2}}-\frac{\ddot{a}}{a}+\frac{\dot{a} \dot{n}}{a n}\right)+\left(\left(\frac{a^{\prime}}{a}\right)^{2}+\frac{a^{\prime} n^{\prime}}{a n}\right)\right]=-\frac{1}{2} b^{2} \Lambda$.

Here a dot is a shorthand for $\partial / \partial t$ and a prime for $\partial / \partial y$. The first four equations correspond to the 00, 04, 11 and 44 components of Einstein's equations. Conservation of the stressenergy tensor gives

$$
\dot{\rho}+3 \frac{\dot{a}}{a}(\rho+p)=0
$$

on each brane. 
We look for static solutions to these equations. One may then reparametrize the fifth coordinate to enforce $b=1$. The resulting equations are most easily solved in terms of the warp factors $A$ and $N$ defined by

$$
a=\exp (A) \quad n=\exp (N) .
$$

For convenience we also introduce $k \equiv \sqrt{-\Lambda / 12}$. In the bulk Einstein equations reduce to

$$
\begin{aligned}
A^{\prime \prime}+2 A^{\prime 2} & =2 k^{2}, \\
2 A^{\prime \prime}+3 A^{\prime 2}+2 A^{\prime} N^{\prime}+N^{\prime \prime}+N^{\prime 2} & =6 k^{2}, \\
A^{\prime 2}+A^{\prime} N^{\prime} & =2 k^{2} .
\end{aligned}
$$

The solution is straightforward. The metric in the bulk is given, for $y \geq 0$, by

$$
\begin{aligned}
a^{2} & =a_{*}^{2} \cosh \left[2 k\left(y-y_{*}\right)\right], \\
n^{2} & =n_{*}^{2} \sinh \left[2 k\left(y-y_{*}\right)\right] \tanh \left[2 k\left(y-y_{*}\right)\right], \\
b^{2} & =1
\end{aligned}
$$

where $y_{*}, n_{*}$ and $a_{*}$ are constants. For related bulk solutions see Ref. [10].

To complete the solution we must examine the field equations at the brane. The delta functions on the right hand side of Eqs. (2.6) and (2.8) must be saturated on the right hand side by the second derivatives. To this effect we demand

$$
\begin{aligned}
\left.2 A^{\prime}\right|_{y=0+} & =-\frac{1}{6} V_{0}-\frac{1}{3} \rho_{0}, \\
-\left.2 A^{\prime}\right|_{y=y_{c}-} & =-\frac{1}{6} V_{c}-\frac{1}{3} \rho_{c}, \\
\left.2\left(2 A^{\prime}+N^{\prime}\right)\right|_{y=0+} & =-\frac{1}{2} V_{0}+p_{0}, \\
-\left.2\left(2 A^{\prime}+N^{\prime}\right)\right|_{y=y_{c}-} & =-\frac{1}{2} V_{c}+p_{c} .
\end{aligned}
$$

The solution to these are two equations of fine tuning,

$$
144 k^{2}=\left(V_{0}+2 \rho_{0}\right)\left(V_{0}-\rho_{0}-3 p_{0}\right)=\left(V_{c}+2 \rho_{c}\right)\left(V_{c}-\rho_{c}-3 p_{c}\right),
$$

and two equations fixing the parameters $y_{*}$ and $y_{c}$ :

$$
12 k \tanh \left[2 k y_{*}\right]=V_{0}+2 \rho_{0}, \quad 12 k \tanh \left[2 k\left(y_{c}-y_{*}\right)\right]=V_{c}+2 \rho_{c} .
$$

The first two conditions, Eq. (2.22) are similar in spirit to the two fine tuning equations in the RS model that set the square of the tension in each brane in a fixed proportion to the bulk cosmological constant. By comparison, the stabilization mechanism of Refs. [2, 11] requires only one fine tuning, which is equivalent to setting the cosmological constant to zero. The last two equations describe what was alluded to in the introduction, that in order for the warp factor to jump by the appropriate amount the location of the brane must be 
chosen accordingly. Hence the radius is stabilized, or, should we say, equilibrated (stability is investigated in the next section).

Note that positive tension branes $(V>0)$ can only exist for $p \leq-\rho$. If $\frac{1}{3} \rho>p>-\frac{1}{3} \rho$ on both branes, solutions exist only for $V_{0}<-12 k$ and $V_{c}<-12 k$. But if $p>\frac{1}{3} \rho, V$ can be larger or smaller than $-12 k$.

If we insist, as in the RS model, that $V_{0}^{2}=V_{c}^{2}=144 k^{2}$ then the conditions (2.22) are identical to the static solution conditions in Eq. (1.2). We recover the RS model taking vanishing matter density and pressure and the limit $y_{*} \rightarrow \infty$.

However we need not insist on imposing $V_{0}^{2}=V_{c}^{2}=144 k^{2}$. In fact, we are now free to chose any tension provided the conditions (2.22) are satisfied and a solution to Eqs. (2.23) with $y_{c}>0$ can be found.

\section{SMALL PERTURBATIONS}

Armed with the new solutions with static matter density, we proceed to investigate the time dependence of small matter perturbations. Let us denote the static solution of the previous section by $n_{0}=e_{0}^{N}, a_{0}=e_{0}^{A}$, and $b_{0}=1$. We look for solutions to the field equations, Eqs. (2.6)-(2.9), of the form

$$
\begin{aligned}
n & =n_{0}(1+\delta n), \\
a & =a_{0}(1+\delta a), \\
b & =b_{0}(1+\delta b) .
\end{aligned}
$$

In addition we set the density on the branes to $\rho_{0}+\delta \rho_{0}$ and $\rho_{c}+\delta \rho_{c}$ and the pressure to $p_{0}+\delta p_{0}$ and $p_{c}+\delta p_{c}$.

We count orders of the perturbative expansion parametrically in $\delta \rho$ and $\delta p$. That is, we re-scale $\delta \rho \rightarrow \epsilon \delta \rho$, count powers of $\epsilon$ and set $\epsilon=1$ at the end of the calculation. In particular this implies that we make no assumption as to the relative importance of temporal or spatial derivatives [6].

To derive the linearized equations in the bulk, we use the parameterization in Eqs. (3.1). The 00, 04, 11 and 44 components of Einstein's equations give

$$
\begin{aligned}
\delta a^{\prime \prime}+A_{0}^{\prime}\left(4 \delta a^{\prime}-\delta b^{\prime}\right) & =4 k^{2} \delta b, \\
\frac{\partial}{\partial t}\left(\left(N_{0}^{\prime}-A_{0}^{\prime}\right) \delta a+A_{0}^{\prime} \delta b-\delta a^{\prime}\right) & =0, \\
2 \delta a^{\prime \prime}+\delta n^{\prime \prime}+\left(6 A_{0}^{\prime}+2 N_{0}^{\prime}\right) \delta a^{\prime}+2\left(A_{0}^{\prime}+N_{0}^{\prime}\right) \delta n^{\prime} & \\
-\left(2 A_{0}^{\prime}+N_{0}^{\prime}\right) \delta b^{\prime}-\frac{1}{n_{0}^{2}}(2 \delta \ddot{a}+\delta \ddot{b}) & =12 k^{2} \delta b, \\
\left(2 A_{0}^{\prime}+N_{0}^{\prime}\right) \delta a^{\prime}+A_{0}^{\prime} \delta n^{\prime}-\frac{1}{n_{0}^{2}} \delta \ddot{a} & =4 k^{2} \delta b .
\end{aligned}
$$

The solution to these equations gives $\delta b$ and $\delta n^{\prime}$ in terms of $\delta a$ :

$$
\begin{aligned}
\delta b & =\frac{1}{A_{0}^{\prime}}\left[F+\delta a^{\prime}+\left(A_{0}^{\prime}-N_{0}^{\prime}\right) \delta a\right], \\
\delta n^{\prime} & =\frac{1}{A_{0}^{\prime}}\left[\frac{1}{n_{0}^{2}} \delta \ddot{a}-\left(2 A_{0}^{\prime}+N_{0}^{\prime}\right) \delta a^{\prime}+4 k^{2} \delta b\right] .
\end{aligned}
$$


In Eq. (3.7) $\delta b$ is understood as shorthand for the solution of Eq. (3.6). In Eq. (3.6) $F$ is a function of $y$ satisfying

$$
F^{\prime}-\frac{A_{0}^{\prime \prime}-4 k^{2}}{A_{0}^{\prime}} F=0 .
$$

It must be observed that $F$ may be discontinuous at $y=0$. In fact, continuity of $\delta b$ at $y=0$ requires $F(0+)+F(0-)=0$. The solution to Eq. (3.8) is $F(y)=F_{*} / \sinh \left(4 k\left(y-y_{*}\right)\right)$, with $F_{*}$ a constant.

We connect the bulk solutions for $y>0$ and $y<0$ demanding continuity of the fields at the brane, $y=0$, and using the jump equations for the discontinuous derivatives at $y=0$. The latter give jump conditions for the perturbations

$$
\begin{aligned}
\left.\delta a^{\prime}\right|_{0+} & =-\frac{1}{6}\left[\left(\frac{1}{2} V_{0}+\rho_{0}\right) \delta b+\delta \rho_{0}\right], \\
\left.\left(2 \delta a^{\prime}+\delta n^{\prime}\right)\right|_{0+} & =\frac{1}{2}\left[\left(-\frac{1}{2} V_{0}+p_{0}\right) \delta b+\delta p_{0}\right] .
\end{aligned}
$$

Similarly, the jump equations at the second brane are

$$
\begin{aligned}
-\left.\delta a^{\prime}\right|_{y_{c}-} & =-\frac{1}{6}\left[\left(\frac{1}{2} V_{c}+\rho_{c}\right) \delta b+\delta \rho_{c}\right], \\
-\left.\left(2 \delta a^{\prime}+\delta n^{\prime}\right)\right|_{y_{c}-} & =\frac{1}{2}\left[\left(-\frac{1}{2} V_{c}+p_{c}\right) \delta b+\delta p_{c}\right] .
\end{aligned}
$$

In addition, conservation of energy gives, on the brane,

$$
\delta \rho_{0}+\left.3\left(\rho_{0}+p_{0}\right) \delta a\right|_{y=0}=0 \quad \text { and } \quad \delta \rho_{c}+\left.3\left(\rho_{c}+p_{c}\right) \delta a\right|_{y=y_{c}}=0 .
$$

The right hand side of these equations could be a non-zero constant. However, we set it to zero since we are not interested in constant shifts in the mass density (since these are accounted for in the exact solution of Sec. [I]).

The jump equations at $y=0$, Eqs. (3.9)-(3.10), determine $\delta a^{\prime}$ in terms of $\delta a$ and give an equation for $\delta a$, namely

$$
\frac{1}{n_{0}^{2} N_{0}^{\prime}} \delta \ddot{a}+\frac{1}{2} \frac{A_{0}^{\prime}}{N_{0}^{\prime}} \delta p_{0}-\frac{1}{6} \delta \rho_{0}=0
$$

The time dependence of the matter is fixed by the continuity equation, Eq. (3.13). Given an equation of state for the perturbations, $\delta p_{0} / \delta \rho_{0}=w_{0}$, one can solve this equation. Let's rewrite the equation as

$$
\delta \ddot{a}-\Gamma_{0}^{2} \delta a=0 .
$$

Then the coefficient $\Gamma_{0}^{2}$ can be expressed in terms of the initial parameters. In terms of $t_{0} \equiv\left(V_{0}+2 \rho_{0}\right) / 12 k=\tanh \left(2 k y_{*}\right)$ we find

$$
\Gamma_{0}^{2}=2 k^{2} n^{2}(y=0)\left[\frac{2}{t_{0}}-\left(3 \omega_{0}+1\right) t_{0}\right]\left[\frac{1}{t_{0}}-t_{0}\right]
$$


Hence, $\Gamma_{0}^{2}>0$ for equations of state with $w_{0}<1 / 3$. But for $w_{0}>1 / 3 \Gamma_{0}^{2}$ can be either positive or negative depending on the value of $t_{0}$ with $\omega_{0}=\frac{1}{3}\left(\frac{2}{t_{0}^{2}}-1\right)$ being the dividing line.

We see that the time dependence of $\delta a$ on the $y=0$ brane is exponential for $\omega_{0}<1 / 3$, but can be oscillatory for $\omega_{0}>1 / 3$ :

$$
\left.\delta a\right|_{y=0}=c_{0} e^{\Gamma_{0} t}+d_{0} e^{-\Gamma_{0} t}
$$

where $\Gamma_{0}$ is either real or purely imaginary. Substituting this solution in the equation for $\delta a^{\prime}$ gives

$$
\left.\delta a^{\prime}\right|_{y=0}=0
$$

This implies that the jump in $F$ vanishes. Therefore $F(y)=0$.

An entirely analogous solution is obtained on the brane at $y=y_{c}$ by replacing the subscript "0" in Eqs. (3.15)-(3.17) by the second brane subscript "c."

To extend the solution on the branes into the bulk we must fix the gauge. There is a gauge freedom, that is, reparametrization invariance consistent with the form of our metric. Starting from the metric

$$
d s^{2}=n^{2}\left(t^{\prime}, y^{\prime}\right) d t^{\prime 2}-a^{2}\left(t^{\prime}, y^{\prime}\right) d \vec{x}^{2}-b^{2}\left(t^{\prime}, y^{\prime}\right) d y^{\prime 2},
$$

we look for infinitesimal transformations

$$
\begin{aligned}
t^{\prime} & =t+T(t, y) \\
y^{\prime} & =y+Y(t, y)
\end{aligned}
$$

that leave the form of the metric invariant and has fixed points at at $y=0$ and $y=y_{c}$. Here $T$ and $Y$ are infinitesimal. The only constraints on these functions come from the absence of off-diagonal terms in the metric,

$$
n^{2} T^{\prime}-b^{2} \dot{Y}=0
$$

and from the fixed points,

$$
Y(t, 0)=Y\left(t, y_{c}\right)=0
$$

Under the gauge transformation the metric variations are

$$
\begin{aligned}
\Delta \delta n & =N_{0}^{\prime} Y+\dot{T} \\
\Delta \delta a & =A_{0}^{\prime} Y, \\
\Delta \delta b & =Y^{\prime} .
\end{aligned}
$$

For simplicity we have indicated the variation about a static solution with $b_{0}=1$ and $\dot{a}_{0}=\dot{n}_{0}=0$. We would like to use this gauge freedom to impose the gauge condition

$$
\delta b(y, t)=0 .
$$


However, this cannot be done in general since there is only one constant of integration in the gauge condition (3.26). Instead consider covering the space with two different patches. We label the metric perturbations $\delta a_{1}, \delta n_{1}$ and $\delta b_{1}$ on the patch defined by $0 \leq y<y_{c} / 2+\epsilon$ and by $\delta a_{2}, \delta n_{2}$ and $\delta b_{2}$ on the patch defined by $y_{c} / 2-\epsilon<y \leq y_{c}$. Given a solution with metric $\delta a, \delta n$ and $\delta b$ we choose the new coordinates by choosing

$$
Y_{1}=-\int_{0}^{y} \delta b(\hat{y}, t) d \hat{y} \quad \text { and } \quad Y_{2}=-\int_{y_{c}}^{y} \delta b(\hat{y}, t) d \hat{y}
$$

Thus $\delta b_{1}=0$ for $0 \leq y \leq y_{c} / 2+\epsilon$ and $\delta b_{2}=0$ for $y \geq y_{c} / 2-\epsilon$.

The solution for $\delta a_{1}$ and $\delta a_{2}$ follows immediately from Eq. (3.6) setting $\delta b=0$ :

$$
\delta a_{1}(y, t)=F_{* 1} / 4 k+A_{0}^{\prime} \xi_{1}(t) \quad \text { and } \quad \delta a_{2}(y, t)=F_{* 2} / 4 k+A_{0}^{\prime} \xi_{2}(t),
$$

where $\xi_{1,2}$ are arbitrary functions of $t$ but independent of $y$. We can now use our jump conditions to specify these completely:

$$
\delta a_{1}(y, t)=\left.\frac{A_{0}^{\prime}(y)}{A_{0}^{\prime}(0+)} \delta a\right|_{y=0+} \quad \text { and } \quad \delta a_{2}(y, t)=\left.\frac{A_{0}^{\prime}(y)}{A_{0}^{\prime}\left(y_{c}-\right)} \delta a\right|_{y=y_{c}-} .
$$

In the overlap region, $\left|y-y_{c} / 2\right|<\epsilon$, these solutions are related by a gauge transformation:

$$
\delta a_{2}-\delta a_{1}=A_{0}^{\prime} \int_{0}^{y_{c}} \delta b(\hat{y}, t) d \hat{y}
$$

Thus we obtain

$$
\begin{aligned}
\int_{0}^{y_{c}} \delta b(\hat{y}, t) d \hat{y} & =\left.\frac{\delta a}{A_{0}^{\prime}}\right|_{y=y_{c}-}-\left.\frac{\delta a}{A_{0}^{\prime}}\right|_{y=0+} \\
& =\frac{c_{c} e^{\Gamma_{c} t}+d_{c} e^{-\Gamma_{c} t}}{k \tanh \left(2 k\left(y_{c}-y_{*}\right)\right)}+\frac{c_{0} e^{\Gamma_{0} t}+d_{0} e^{-\Gamma_{0} t}}{k \tanh \left(2 k y_{*}\right)}
\end{aligned}
$$

where it is understood that $\Gamma_{0, c}$ can be purely imaginary if $\omega_{0, c}>\frac{1}{3}$.

\section{DISCUSSION AND CONCLUSIONS}

In Sec. II we gave a class of solutions to the field equations with matter on the two branes. The solutions are static. The price to pay for time independence is a fine tuning of the amount of energy on each brane, as expressed in Eq. (2.22). These fine tunings are no worse than the corresponding fine tunings in the RS model.

The salient feature of the solutions found in Sec. II is that the physical size of the fifth dimension is fixed. This suggested the exciting possibility that the radion stabilization problem is not an issue in models with matter on the branes.

However, static solutions are not cosmologically acceptable. Nevertheless, one wonders if even for non-static solutions with matter the radius is stabilized. Short of finding an exact non-static solution we have exhibited in Sec. III an approximate solution by linearizing the field equations around the static solutions. 
The result of the linearized analysis is summarized by Eqs. (3.17) and (3.32). The first one establishes that the metric perturbations have exponential time dependence for equations of state with $\omega=p / \rho<1 / 3$, but can be oscillatory for $\omega>1 / 3$. The second gives the time dependence of the radius of the space. Indeed, a measure of the radius is

$$
L=\int \sqrt{-G_{M N} d x^{M} d x^{N}}
$$

along a line of constant $t$ and $\vec{x}$ :

$$
L=y_{c}+\int_{0}^{y_{c}} \delta b(\hat{y}, t) d \hat{y}
$$

For $\omega<1 / 3$ the radius grows exponentially, at least while the exponential is small enough that the linearized solution remains a good approximation. On the other hand, for $\omega>1 / 3$ the radius can oscillate about the equilibrium value $y_{c}$ or can grow exponentially depending on the value of $t_{0}=\frac{V_{0}+2 \rho_{0}}{12 k}$.

One gains some understanding of the behavior of the scale factor $a$ on the brane by considering the full, non-perturbative description of its evolution at an orbifold fixed point. Since we do not wish to insist that $V^{2}=144 k^{2}$ we modify Eq. (1.2) to allow for an unconstrained tension,

$$
\left(\frac{\dot{a}^{2}}{a^{2}}\right)+\left(\frac{\ddot{a}}{a}\right)=\frac{1}{72} V(\rho-3 p)-\frac{1}{36} \rho(\rho+3 p)+\frac{1}{72} V^{2}-2 k^{2},
$$

Let the equation of state be $p=w \rho$. Using the equation of conservation, Eq. (2.10), one has $\rho=\rho_{0} a_{0}^{3(1+w)} / a^{3(1+w)}$, where $\rho_{0}$ and $a_{0}$ are the density and scale factor at a fixed time. Then one can rewrite the equation for the form factor as the equation for a particle with displacement $r=a^{2}$ in a potential, $\ddot{r}=-U^{\prime}(r)$, with

$$
U(r)=C_{1} r^{2-\frac{3}{2}(1+w)}+C_{2} r^{2-3(1+w)}+C_{3} r^{2}
$$

where

$$
\begin{aligned}
& C_{1}=-\frac{\rho_{0} a_{0}^{3(1+w)} V}{18} \\
& C_{2}=-\frac{\rho_{0}^{2} a_{0}^{6(1+w)}}{18} \\
& C_{3}=2 k^{2}-\frac{V^{2}}{72}
\end{aligned}
$$

Recall that for $\omega>-1$ our solutions must have negative tension branes $V<0$, so $C_{1}>0$ and $C_{2}<0$. For $\frac{1}{3}>\omega>-\frac{1}{3}, V<-12 k$ and, therefore, $C_{3}$ is negative and the only extremum of $U(r)$ is a maximum. But for $w>\frac{1}{3}$, V can be larger or smaller than $-12 k$ and, therefore, the sign of $C_{3}$ can be either positive or negative. The extremum of $U(r)$ can, therefore, be a maximum or minimum, in accordance with our analysis of small perturbations.

The physical interpretation of the solutions discussed here is not straightforward. One cannot, as in the case of the RS model, simply renormalize fields on either brane to cast 
their kinetic energy term in standard form, since $n \neq a$. Moreover, a null trajectory parallel to the branes has $d x / d t=n / a$. Since $n / a=\left(n_{*} / a_{*}\right) \tanh \left[2 k\left(y-y_{*}\right)\right]$ differs between the two fixed points, one can arrange for superluminal signal travel. For example, on one brane one can send a graviton across to the other brane, relay the signal along the brane by photons, and then relay the signal back to the first brane via gravitons. Neglecting the time of travel between branes, we see that the interval between emission and reception can be shorter than the time for a photon to travel between the same two points directly on the first brane [12]. A complete discussion is the subject of a forthcoming paper.

Since for standard fluids in equilibrium the equation of state must have $\omega<1 / 3$, the exponential growth of the scale factor and radius make this model an unlikely candidate for cosmology. However, it may be possible to stabilize the radius by modifying the model, say, by adding matter in the bulk, e.g., scalar fields [2]. The cosmology of such a model may indeed be acceptable [6].

Note Added While this article was being completed three related works have been submitted to the archives. Ref. [13] discusses general solutions with different space and time components of the 5-d metric. We have not attempted to check if an explicit coordinate transformation can convert our bulk solution to a form used in Ref. [13. The authors also discuss Lorentz symmetry violations due to different propagation speeds of bulk and brane fields. Ref. [14] uses supersymmetric gauge dynamics for stabilizing the radius and Ref. [15] uses the Casmir force due to a bulk scalar field.

Acknowledgments The work of B.G. is supported by the U.S. Department of Energy under contract No. DOE-FG03-97ER40546, the work of D.N. under contract No. DEFG02-90ER40542, and the work of W.S. under cooperative research agreement DE-FC0294ER40818. 


\section{REFERENCES}

[1] L. Randall and R. Sundrum, Phys. Rev. Lett. 83, 3370 (1999) hep-ph/9905221.

[2] W. D. Goldberger and M. B. Wise, Phys. Rev. Lett. 83, 4922 (1999) [hep-ph/9907447.

[3] L. Mersini, "Radion potential and brane dynamics," hep-ph/0001017; H. B. Kim, Phys. Lett. B478, 285 (2000) hep-th/0001209]; J. Garriga O. Pujolas and T. Tanaka, "Radion effective potential in the brane-world," hep-th/0004109; U. Gunther and A. Zhuk, "A note on dynamical stabilization of internal spaces in multidimensional cosmology," hep-ph/0006283; W. D. Goldberger and I. Z. Rothstein, Phys. Lett. B491, 339 (2000) hep-th/0007065; I. Brevik, K. A. Milton, S. Nojiri and S. D. Odintsov, "Quantum (in)stability of a brane-world AdS(5) universe at nonzero temperature," hep-th/0010205.

[4] P. Binetruy, C. Deffayet and D. Langlois, Nucl. Phys. B565, 269 (2000) hepth/9905012.

[5] C. Csaki, M. Graesser, C. Kolda and J. Terning, Phys. Lett. B462, 34 (1999) hep-ph/9906513; J. M. Cline, C. Grojean and G. Servant, Phys. Rev. Lett. 83, 4245 (1999) hep-ph/9906523; D. J. Chung and K. Freese, Phys. Rev. D 61, 023511 (2000) hep-ph/9906542; P. Binetruy, C. Deffayet, U. Ellwanger, and D. Langlois, Phys. Lett. B477, 285 (2000) hep-th/9910219]; E. E. Flanagan, S. H. Tye and I. Wasserman, Phys. Rev. D62, 044039 (2000) hep-ph/9910498; H. Stoica, S. H. Tye, and I. Wasserman, Phys. Lett. B482, 205 (2000) [hep-th/0004126]; R. N. Mohapatra, A. Perez-Lorenzana and C. A. de Sousa Pires, "Cosmology of brane-bulk models in five dimensions," hep$\mathrm{ph} / 0003328$.

[6] C. Csaki, M. Graesser, L. Randall and J. Terning, Phys. Rev. D62, 045015 (2000) hep-ph/9911406;

[7] J. M. Cline and H. Firouzjahi, Phys. Lett. B495, 271 (2000) hep-th/0008185.

[8] B. Grinstein, D. Nolte and W. Skiba, Phys. Rev. D62, 086006 (2000) hep-th/0005001.

[9] B. Grinstein, D. Nolte and W. Skiba, "On a covariant determination of mass scales in warped backgrounds," hep-th/0012074.

[10] S. H. H. Tye and I. Wasserman, "A brane world solution to the cosmological constant problem," hep-th/0006068; E. Flanagan, N. Jones, H. Stoica, S. H. H. Tye and I. Wasserman, "A Brane World Perspective on the Cosmological Constant and the Hierarchy Problems," hep-th/0012129.

[11] O. DeWolfe, D. Z. Freedman, S. S. Gubser and A. Karch, Phys. Rev. D62 (2000) 046008 hep-th/9909134.

[12] D. J. Chung and K. Freese, Phys. Rev. D 62, 063513 (2000) hep-ph/9910235.

[13] C. Csaki, J. Erlich and C. Grojean, "Gravitational Lorentz Violations and Adjustment of the Cosmological Constant in Asymmetrically Warped Spacetimes," hep-th/0012143.

[14] M. Luty and R. Sundrum, "Hierarchy Stabilization in Warped Supersymmetry," hepth/0012158.

[15] R. Hofmann, P. Kanti and M. Pospelov, "(De-)Stabilization of an extra dimension due to a Casimir force," hep-ph/0012213. 